\title{
Activités
}

\section{Écrits de relève de poste : une activité continue}

Shift-changeover logs: an ongoing activity

Valérie Le Bris et Béatrice Barthe

\section{QpenEdition}

\section{Journals}

Édition électronique

URL : https://journals.openedition.org/activites/532

DOI : 10.4000/activites.532

ISSN : $1765-2723$

\section{Éditeur}

ARPACT - Association Recherches et Pratiques sur les ACTivités

Référence électronique

Valérie Le Bris et Béatrice Barthe, «Écrits de relève de poste : une activité continue », Activités [En ligne], 10-1 | Avril 2013, mis en ligne le 15 avril 2013, consulté le 21 septembre 2021. URL : http:// journals.openedition.org/activites/532 ; DOI : https://doi.org/10.4000/activites.532

\section{(2) $\odot \Theta \Theta$}

Activités est mis à disposition selon les termes de la licence Creative Commons Attribution - Pas d'Utilisation Commerciale - Pas de Modification 4.0 International. 


\title{
Écrits de relève de poste : une activité continue
}

\author{
Valérie Le Bris
}

ergonova, Conseil, Étude \& Formation en ergonomie, 78 Chemin des 7 Deniers, 31200 Toulouse

v.lebris@ergonova.fr

\section{Béatrice Barthe}

Université Toulouse 2 le Mirail, Laboratoire CLL“" -LTC, UMR 5263 CNRS, Maison de la Recherche, 5 allée Antonio Machado, 31058 Toulouse Cedex 9

beatrice.barthe@univ-tlse2.fr

\section{SUMMARY}

Shift-changeover logs: an ongoing activity. Shift changeover periods, when one team relieves another, contribute to achieving the continuity and reliability of shift work. This article considers first-line supervisory staff shift changeovers to be an ongoing activity carried out across successive shifts. The study looks at supervisory activity in the context of a final aircraft assembly line running on a $2 \times 8$ discontinuous schedule where changeovers alternate between shift changes where the two teams meet, allowing for oral and written exchanges, and changeovers where they do not meet and only written exchanges are used. The analysis focuses on the activities of writing and using written handovers throughout shift work (before, during, and after shift changeovers). It showed, firstly, that written handovers were prepared throughout the shift no matter what type of shift changeover would follow, but were systematic whenever the upcoming changeover was without meeting, and secondly, that written handovers about what the incoming operator had to do, and what the outgoing operator had done, acted as supports for and records of collective-activity coordination. These results allow us to see shift changeovers as a task that overarches other first-line supervisory tasks and as contributing to work coordination across shifts. Some principles are proposed for adjusting and optimising shift changeovers.

\section{K" Y WORDS}

atypical schedules, supervisor, shift work, communications, collective work

La présente étude s'inscrit dans une réflexion sur les horaires de travail instruite par un constructeur aéronautique. Les horaires de certaines chaines d'assemblage final d'avions avaient été rallongés et aménagés dans le but d'absorber les augmentations de cadence. L'organisation horaire mise en place était alors très inégale du point de vue des temps de chevauchement d'équipes successives (allant de plus d'une heure à aucun chevauchement selon les vacations), posant ainsi la question du rôle des relèves de poste. L'industriel a donc souhaité réorienter la réflexion sur les relèves de poste afin de les outiller.

L'importance de la relève de poste pour la fiabilité des systèmes est tristement établie au travers de rapports d'incident ou d'accident qui mettent en cause une mauvaise communication entre les différents postes qui se sont succédés (pollution radioactive à Sellafield Beach cité par Lardner, 1996 ; crash du Continental “ xpress Flight 257, National 
Transportation Safety Board, 1992). Même si les conséquences de ces défaillances sont souvent moins spectaculaires et dramatiques dans les situations de production manufacturière, les transmissions entre les postes demeurent cruciales, que ce soit pour la bonne utilisation des moyens de production, pour une réalisation appropriée du travail ou pour l'assurance de la continuité de production dans des contraintes de délais, de coût, de qualité et de sécurité.

Cela d'autant plus que l'activité d'assemblage aéronautique a pour principales caractéristiques : une fabrication de petites séries avec un produit hautement «customisé »; des cycles d'assemblage longs (un avion reste plusieurs jours sur une même station); un grand nombre d'opérations à réaliser pendant le cycle (environ 40 à 120 opérations réparties sur plusieurs zones de l'avion) dont la durée de réalisation varie de l'heure à plusieurs vacations. C'est pour ces raisons que la relève de poste a été analysée du point de vue des opérateurs qui supervisent une station : l'encadrement de proximité.

La relève de poste est ici considérée comme un objet d'étude à part entière, investigué pour comprendre les processus spécifiques qui permettent une prise de relais optimale entre les différentes équipes qui se succèdent en situation de travail posté. L'objectif est de produire des connaissances pour l'aménagement de la relève de poste, notamment ici des écrits participants à la relève de poste, et ce, à partir d'une analyse ergonomique du travail de l'encadrement de proximité des chaînes d'assemblage dans le secteur aéronautique (le lecteur intéressé pourra se référer à l'intégralité de l'étude, Le Bris, 2010).

\section{1.- La relève de poste de l'encadrement de proximité}

\section{1.- Définitions et positionnement}

Généralement, la relève de poste est définie comme la période de remplacement d'une équipe de travail par une autre, dans les situations de travail fonctionnant en horaires postés (Grusenmeyer, 1991). Lorsque les modes de fonctionnement de ces horaires sont discontinus, c'est-à-dire avec un arrêt de l'activité la nuit et les week-ends (Quéinnec, Teiger, \& de Terssac, 2008), la communication entre les équipes successives ne peut alors être que médiatisée, via par exemple un cahier de transmission. Peuvent alors être distinguées: des relèves avec rencontre entre les opérateurs montants et descendants, caractérisées par la possibilité d'activités verbales orales directes; et des relèves sans rencontre, caractérisées par des transmissions verbales écrites laissées à l'attention de l'équipe suivante. Les avantages de la communication en face à face des premières relèves sur les secondes ont été à maintes reprises mis en évidence (Gramopadhye, Kelbar \& Watson, 1999 ; Parke \& Kanki, 2008 ; Wilson, Galliers, \& Fone, 2005). Nous avons aussi montré l'importance de ces échanges oraux lors de la rencontre, notamment pour l'actualisation des représentations entre les opérateurs montants et descendants (Le Bris, Barthe, Marquié, Kerguelen, Aubert, et al., 2012).

Lorsqu'il y a rencontre entre les deux équipes qui se relaient, les auteurs s'accordent à considérer qu'une relève de poste se déroule en plusieurs phases : la préparation, la rencontre et la prise de poste (Cheung, Kelly, Beach, Berkeley, Bitterman, et al., 2010 ; Davillerd \& Grusenmeyer, 1993 ; Lardner, 1996 ; Perry, 2004). Cette caractérisation soulève deux contradictions : les relèves sans rencontre ne sont pas prises en compte alors qu'elles sont quotidiennes dans le travail en horaires discontinus ; et la relève de poste est définie comme un moment à part durant lequel l'activité au et du poste serait suspendue, or ce n'est pas toujours le cas. Dans les situations de gestion de processus dynamiques par exemple, les opérateurs poursuivent la réalisation de leur activité de travail pendant la phase de la rencontre. L'inverse existe aussi, car annoter un dossier au crayon de papier, coller un postit, rédiger une information dans un calepin rangé dans la poche de sa blouse (Hardey, Payne, $\&$ Coleman, 2000) sont autant de pratiques «pour soi» (Moatty, Rouard, \& Teiger, 2004) qui interviennent dans le cours de l'activité «normale» de la vacation et qui permettent de 
capitaliser l'information notamment pour la relève de poste suivante. L'analyse de ce qui se passe «en dehors» de la rencontre nécessite alors d'adopter une démarche de compréhension de l'activité de travail dans sa continuité, c'est-à-dire au cours des postes qui se succèdent.

L'objet de cet article est de considérer la relève de poste non pas comme un moment limité dans le temps durant lequel se déroulerait une activité particulière de relève, et ce en marge de l'activité dite «normale » du poste, mais plutôt comme une activité inscrite dans l'activité de travail mise en œuvre au cours des vacations qui se succèdent.

\section{2.- Des supports de transmissions pour la relève de poste}

La communication entre deux équipes qui se succèdent est soutenue par deux types de supports: ceux dédiés aux transmissions de relève de poste; et ceux qui sont non spécifiques, mais instrumentalisés au sens de Rabardel (1995) lors des relèves de poste, c'est-à-dire qui renvoient à l'ensemble des objets du travail (dossier des patients, fiches suiveuses, gamme de travail, e-mails, pièce défectueuse, etc.).

Les supports dédiés aux transmissions tels que les journaux de bord, les cahiers de liaison ou les enregistrements audio ont pour spécificités : d'engendrer des transmissions asynchrones, les feedbacks ne pouvant intervenir que d'une relève de poste sur l'autre; de mettre en œuvre des processus cognitifs tels que la planification, la formulation, la révision et l'exécution graphomotrice (Hayes \& Flower, 1980) coûteux en ressources de mémoire de travail (Kellogg, 1996). De plus, le transfert d'informations est dépendant du modèle de l'interlocuteur (Cahour \& Falzon, 1991) détenu par l'opérateur descendant sur l'opérateur montant.

La formalisation de ces supports n'est pas systématique bien que cela présente des avantages pour fiabiliser la transmission. À l'hôpital, le développement des transmissions écrites ciblées structure leur préparation avec une centration sur les problèmes du jour du patient à travers un raisonnement clinique (par exemple le format Données-Actions-Résultats étudié par Boucheix et Coiron (2008). Les questions de l'historique et de l'évolution d'un problème ont aussi été retenues lors de l'informatisation des cahiers de quart dans une centrale nucléaire française dans laquelle nous avons été invitées à suivre un changement d'équipes. Les cahiers de quart sont scindés en plusieurs parties relatives aux différents domaines de l'activité des opérateurs (par exemple réactivité, refroidissement, confinement, etc.). Cette rédaction est horodatée. De vacation en vacation, jusqu'à la résolution du problème, les informations rédigées antérieurement sont reprises automatiquement et l'opérateur rajoute les évènements qu'il a rencontrés dans sa vacation. Cette structure permet aux opérateurs de balayer les différents domaines de leur activité de travail et de garder l'historique «à portée de main » sans toutefois prendre le risque de recopiages malheureux comme dans le cas de la contamination nucléaire de la mer du Nord à Sellafield Beach (novembre 1983, cité par Lardner, 1996).

Les atouts des supports de transmissions écrites sont de laisser une trace durable dans la situation de travail et d'être consultables par l'ensemble des acteurs présents aux différents moments.

\section{3.- Écrire pour mémoriser et transmettre}

Les transmissions écrites participent à assurer la continuité et la fiabilité d'un processus, car elles sont une trace de l'activité de relève de poste. Ce sont des écrits sédentaires (Delcambre, 1993, 1997), c'est-à-dire que les informations véhiculées restent dans la situation de travail une fois que l'opérateur descendant est parti. Dans le cas d'une relève sans rencontre, la constitution d'une telle trace s'impose par l'impossibilité de communiquer oralement. Dans le cas d'une relève avec rencontre, elle devient moins nécessaire.

". $\mathrm{n}$ fonction des supports utilisés, le contenu des transmissions est soit un résumé, soit une liste d'évènements saillants de la vacation qui s'achève. Si la préparation de la relève, c'est- 
à-dire la rédaction des écrits avant la rencontre ou la fin de poste, intervient en toute fin de vacation, cela demande à l'opérateur un effort cognitif important. Ainsi, les opérateurs font appel à leur mémoire à long terme et plus particulièrement à leur mémoire épisodique (Craik \& Lockhart, 1972 ; Tulving, 1972) pour se souvenir des évènements qui ont eu lieu pendant la vacation qui vient de s'écouler. La facilité ou la difficulté pour recouvrer ultérieurement une information mémorisée dépend de nombreux facteurs : au moment de l'encodage, elle dépend de l'état chronobiologique (Maury \& Quéinnec, 1993), de l'état émotionnel suscité par l'évènement (D'Argembeau \& Van der Linden, 2006), de la disponibilité liée à des contraintes organisationnelles (ex. : enchaînement de réunions) ou de la tâche dans laquelle l'opérateur est impliqué ; au moment du recouvrement, elle dépend des stratégies spécifiques développées (post-it, etc.). Le recouvrement est une procédure entraînant des oublis ou des déformations. Bisseret (1970) a proposé le concept de mémoire opérationnelle ayant trait aux informations mémorisées se rapportant directement au travail en cours. Sperandio (1975) a testé cette mémoire opérationnelle en condition réelle de travail chez des contrôleurs aériens. Il a montré que l'augmentation de la quantité de données traitées (et donc susceptibles d'être rappelées) et l'allongement de la durée de rétention provoquent une diminution de la rétention des données quand celles-ci sont devenues caduques. Ce caractère de sélectivité opérationnelle qui se manifeste au cours de la réalisation des tâches a ensuite été testé sur un empan temporel plus grand, c'est-à-dire toute une vacation de travail. L'auteur a observé que la rétention des données en fin de vacation de travail était étroitement liée au degré d'importance de chaque donnée élémentaire utilisée au cours de la résolution des problèmes pendant la vacation.

Le recouvrement des informations est modulé par les moyens mis à disposition au cours de la vacation (Boucheix \& Coiron, 2008 ; Grosjean \& Lacoste, 1999), mais également par les exigences du travail, leurs fluctuations au cours du poste, le rythme de travail, les interférences, et plus généralement par les spécificités du métier.

\section{4.- Le travail de l'encadrement de proximité}

L'étude des écrits de relève de poste présentée a été menée auprès de chefs d'équipe d'une chaine de production. Les chefs d'équipe occupent, avec les chefs de chantier, d'atelier, de fabrication, les agents de maîtrise, les contremaîtres, les conducteurs de travaux ce que l'on appelle une fonction d'encadrement dite de proximité.

L'encadrement de proximité de l'industrie gère des opérateurs qui réalisent des opérations de transformation sur un système technique. Cet encadrement a une fonction de management global, intégrant plusieurs domaines d'activité tournés vers la mise en œuvre rapide d'actions et de décisions à rentabilité immédiate (Létondal, 1997). Il a pour mission la gestion du secteur auquel il est affecté. Cette gestion se partage entre: la gestion du processus technique et de sa qualité (suivi de l'avancement et ajustement, animation de la résolution de problèmes techniques); la gestion des équipements techniques (suivi de l'état de fonctionnement des équipements, médiation de la relation entre ses subordonnées et les agents de maintenance) ; la gestion du personnel (temps de travail, formation, promotion); et la gestion de la sécurité sur son secteur (information sur les règles de sécurité, gestion des équipements de protection collective et individuelle) (Mascia, 2001). La variabilité est permanente, partiellement prévisible et contrôlée par les moyens techniques et organisationnels existants. L'encadrement de proximité joue, au quotidien, un rôle important de régulation de la variabilité et de réduction de la complexité, rôle qui peut s'avérer difficile (Mascia, 2001).

L'encadrement de proximité est un maillon important pour la continuité et la cohérence des activités au sein de l'atelier, dont l'activité présente les mêmes caractéristiques que celle des cadres: fragmentation et parcellisation, interactions et déplacements nombreux (Dieumegard, Saury, \& Durand, 2004 ; Hales, 1986 ; Rogalski \& Langa, 1997). Ainsi leur journée se caractérise par: un indéterminisme et un engagement parcellisé sur des problématiques ou des dossiers; un éloignement du bureau où se trouve(nt) bien souvent 
le(s) support(s) de transmission de relève de poste; de nombreuses interruptions et interactions (Bellies \& Beauguil, 2008; Trouvé, 1997; Walker, Guest, \& Turner, 1956). Cela laisse peu de place pour préparer les transmissions de relève de poste et conduit l'encadrement de proximité à reporter dans le temps la rédaction d'une transmission, au risque de ne plus s'en souvenir quand les conditions seront réunies pour réaliser cette tâche. " n effet la charge cognitive qui découle de leur activité, la diversité des problématiques et des informations traitées peuvent compliquer le recouvrement en fin de vacation des informations utiles pour l'opérateur montant, comme l'a montré Sperandio (1975) à propos des contrôleurs aériens.

\section{5.- Différents collectifs de travail à coordonner}

Le fonctionnement en horaires postés implique la succession de plusieurs équipes pour pouvoir atteindre un objectif commun de production, qualité, délais, coûts, sécurité, etc. On distinguera alors des collectifs ${ }^{1}$ de travail synchrones et des collectifs asynchrones.

\subsection{1.- Des collectifs de travail synchrones : immédiats et périphériques}

Lors d'une même vacation de travail, plusieurs collectifs de travail peuvent être distingués en tenant compte des critères de taille, d'éloignement et de métier.

D'une part, le collectif de travail immédiat est constitué à partir des activités collectives mises en œuvre par des opérateurs poursuivant un but commun, travaillant au sein d'une même vacation et partageant un même «objet » de travail (au sens de Barthe \& Quéinnec, 1999). Par exemple, à l'hôpital, ce collectif immédiat rassemble l'ensemble des infirmières, des aides-soignants et des médecins affectés au même service. Ces activités collectives portent aussi bien sur des actions mises en œuvre que sur le partage de représentations (Aubert, 1998 ; Barthe, 2003 ; Benchekroun, 2000 ; Grosjean, 2005 ; Heath \& Luff, 1994 ; Salembier \& Zouinar, 2005 ; Savoyant, 1985). Ce collectif immédiat peut être distribué si l'installation sur laquelle ils travaillent est très grande. Par exemple, dans le cadre de l'exploitation d'une centrale nucléaire, nous considérons que le collectif immédiat comprend les opérateurs en salle de commande, le chef de quart, mais aussi les rondiers. L'espace « partagé » correspond alors à la tranche nucléaire. La notion d'immédiateté est alors relative aux nombreuses interactions des rondiers avec la salle de commande.

D'autre part, le collectif de travail périphérique qui transparaît au travers des activités collectives mises en œuvre par des opérateurs qui travaillent aux mêmes horaires, qui sont physiquement éloignés dans l'espace, et qui partagent ponctuellement au cours de la vacation un objectif commun. Il se rapproche de la notion de réseau (Bourdon \& WeillFassina, 1994 ; Caroly, 2010). Ce collectif se manifeste, par exemple, lors du transfert d'un patient d'un service hospitalier à un autre (Arora, 2005 ; Coleman \& Berenson, 2004 ; Cook, Render, \& Woods, 2000) ; lorsque les agents en salle de contrôle dans un aéroport contactent l'équipe de maintenance de l'aéroport pour résoudre un problème sur une passerelle (Goodwin \& Goodwin, 1997). Ce collectif de travail est moins pérenne que le collectif immédiat.

\subsection{2.- Des collectifs de travail asynchrones : temporellement distribués}

Le collectif de travail temporellement distribué transparaît au travers des activités collectives mises en œuvre par-delà les vacations successives. Il se compose alors de plusieurs équipes de travail qui se succèdent. Les opérateurs mettent en œuvre des activités collectives alors qu'ils réalisent des actions à des moments différents. Leurs activités sont asynchrones. Ce collectif peut s'envisager suivant deux niveaux : au niveau de l'atelier ou du service. Il est

1 Nous parlerons délibérément de « collectif » non pas dans le sens de Caroly et Clot (2004) mais plutôt dans un sens commun, qui se rapproche du terme «équipe effective de travail » (Leplat, 1993) au sein de laquelle les opérateurs sont engagés dans une activité collective de travail (Barthe \& Quéinnec, 1999). 
constitué des deux collectifs précédents (immédiat et périphérique). Au niveau de l'opérateur, il correspond au binôme affecté à un poste de travail (par exemple le conducteur de machine) ou à une fonction définie (par exemple le chef d'équipe). Le fonctionnement de ce collectif de travail particulier est directement lié aux relèves de poste.

\subsection{3.- La relève de poste : un outil de coordination essentiel pour les collectifs asynchrones}

Lorsque plusieurs opérateurs sont engagés dans une activité collective (Leplat, 1993) quel que soit le collectif de travail considéré (immédiat ou périphérique, synchrone ou asynchrone), ils doivent à un moment donné coordonner leurs actions, c'est-à-dire les agencer dans un certain ordre afin d'atteindre le but final de façon efficace.

La relève de poste joue un rôle essentiel dans la coordination des collectifs asynchrones, dans l'articulation des tâches qui seront réalisées par les collectifs de travail. La dimension temporelle (opérations effectuées en simultané ou en différé) et la dimension fonctionnelle (c'est-à-dire le degré de dépendance entre ces opérations) (Leplat \& Savoyant, 1983) constituent deux dimensions clés de la coordination des collectifs asynchrones.

Pour coordonner leurs actions, descendant et montant doivent partager un référentiel commun qui permet l'intercompréhension de la situation, l'accord mutuel sur les actions à entreprendre et sur leur réalisation effective (Giboin, 2004). Il est formé à travers l'ajustement des représentations individuelles en réduisant les écarts entre les représentations individuelles de chaque opérateur impliqué dans l'activité collective (de Terssac \& Chabaud, 1990). C'est ce que montre Grusenmeyer (1995) avec la construction de représentations fonctionnelles partagées sur un dysfonctionnement pendant les communications orales de relève de poste. De plus le partage d'un référentiel commun est nécessaire au montant pour interpréter les transmissions du descendant notamment au cours d'une relève sans rencontre.

Mais la construction et le partage d'un référentiel commun, son ajustement et la coordination qui pourra être mise en œuvre par la suite nécessitent de communiquer. À ce propos, Leplat et Savoyant (1983) distinguent les communications d'orientation générale, préalables à la réalisation de l'action (ce qu'il faut faire), les communications parallèles à l'exécution de l'action et les communications de contrôle qui interviennent après l'action (ce qui a été fait). La relève de poste permet d'assurer cette communication inter-équipes (avant, pendant, après l'action), nécessaire pour la coordination (Grosjean \& Lacoste, 1999). À défaut de rencontre, les transmissions écrites prennent le relais voire supportent la communication orale lors de la rencontre.

\section{6.- Une activité de relève de poste transversale aux autres tâches de l'encadrement de proximité ?}

L'encadrement de proximité joue un rôle important pour gérer la coordination des opérations dans le temps, car il occupe une fonction de pivot à la fois dans l'atelier c'est-à-dire au niveau du collectif immédiat et temporellement distribué, mais aussi à l'interface de l'atelier c'est-à-dire avec le collectif périphérique. Les caractéristiques de son activité (indéterminisme et engagement parcellisé sur des problématiques ou des dossiers, éloignement $\mathrm{du}$ bureau, interruptions et interactions nombreuses, charge cognitive importante) interrogent sur la façon dont il aborde le changement d'équipe dans un contexte horaire où les rencontres ne sont pas systématiquement possibles et sur la place qu'occupe la relève de poste dans son activité.

$\mathrm{Au}$ regard du modèle existant de la relève de poste, nous postulons que celle de l'encadrement de proximité n'est pas un moment circonscrit dans le temps. Ainsi la tâche de transmissions (écrites) fait partie du travail en horaires postés, même si elle n'est pas toujours formalisée. Il s'agit d'une tâche transversale aux autres tâches de l'encadrement de proximité qui participe à la coordination du travail à travers les vacations. Le process étant 
dynamique, la tâche de transmission et de coordination va devoir s'adapter à l'évolution du process. Ce travail de transmission, considéré comme participant à la coordination (ce qui est à faire, ce qui reste à faire) va alors être un support à l'action des opérateurs montants et descendants. Ainsi :

— Les transmissions écrites interviennent à différents moments de la vacation et non pas seulement à la fin de la vacation. Cela demande à l'encadrement de proximité d'être en capacité (temporelle, géographique) de rédiger la transmission au moment, avec le risque que l'information rédigée évolue et nécessite d'être corrigée ;

— La rédaction de transmissions écrites permet de guider l'activité de travail de l'encadrant de proximité montant (planifier ce qui reste à faire, tracer et mémoriser ce qui est fait). Les transmissions écrites constituent un support à l'activité collective de l'encadrement de proximité lors de la rencontre.

\section{2.- La situation de travail}

\section{1.- Les opérations industrielles et éléments d'organisation}

Cette étude s'est déroulée sur une station d'assemblage de structures comportant deux grandes opérations : la jonction des trois tronçons du fuselage de l'avion et le montage de gros éléments (des trains d'atterrissage, des éléments de la queue de l'avion, des mâts réacteurs).

La station étudiée fonctionne suivant un horaire $2 \times 8$, avec une vacation de jour (7h3016h15) et une vacation de soir (16h15-00h30). Les opérateurs disposent de plages flexibles pour prendre et quitter leur poste : entre $7 \mathrm{~h}$ et $8 \mathrm{~h}$ en début de vacation de jour; entre $15 \mathrm{~h} 45$ et $16 \mathrm{~h} 30$ entre la vacation de jour et de soir; et entre $00 \mathrm{~h}$ et $01 \mathrm{~h}$ en fin de vacation de soir. Cette organisation en horaires discontinus implique une relève sans rencontre entre $00 \mathrm{~h} 30$ et $7 \mathrm{~h} 30$ et une relève avec possibilité de rencontre pendant la plage flexible, entre $15 \mathrm{~h} 45$ et $16 \mathrm{~h} 30$.

Cette étude a porté sur l'activité de travail de 4 chefs d'équipe (hommes) : deux affectés aux opérations de jonction et deux affectés aux opérations de montage. Au cours d'une vacation, le chef d'équipe est responsable d'une dizaine d'agents de fabrication. Il est assisté de représentants des métiers technique et logistique, et de représentants du métier qualité.

\section{2.- Les tâches réalisées}

Les chefs d'équipe ont pour tâches de piloter les activités de production et de coordonner l'avancement de l'ensemble des travaux réalisés en respectant les objectifs de coûts, qualité et délais ainsi que de sécurité. De plus, ils assurent la gestion des agents de fabrication (présence, carrière, formation, etc.) et des moyens industriels (métrologie des outils, etc.). L'activité mise en œuvre par les chefs d'équipe pour éviter toute rupture de la production relève de l'anticipation et de la récupération quotidienne d'aléas.

Il n'y a pas de tâche spécifique pour gérer la continuité, c'est implicite. La continuité des activités de production n'est considérée qu'à travers l'objectif de gestion des opérations d'assemblage et des dysfonctionnements afférents au cours des vacations. La gestion de la continuité demeure à la discrétion des opérateurs qui se relèvent. L'appropriation de cette tâche s'est traduite par la mise en place d'un cahier de liaison dans lequel les chefs d'équipe dressent un état d'avancement des opérations réalisées et restant à réaliser sur les différentes zones de travail de l'avion en cours, un état d'avancement des dossiers relatifs au traitement d'aléas techniques (défaut de réalisation par exemple) ou logistiques (défaut d'approvisionnement par exemple), et un état d'avancement des opérations en reliquat sur des avions antérieurs. 


\section{3.- Le cahier de liaison}

Le cahier de liaison des chefs d'équipe est un cahier de format A4, à petits carreaux et spiralé, sans structure spécifique de rédaction (cf. Figure 1). Il reste posé sur leur bureau, bien en évidence et donc accessible à tous. Ce sont les chefs d'équipe qui gèrent par la suite son archivage ou sa destruction.

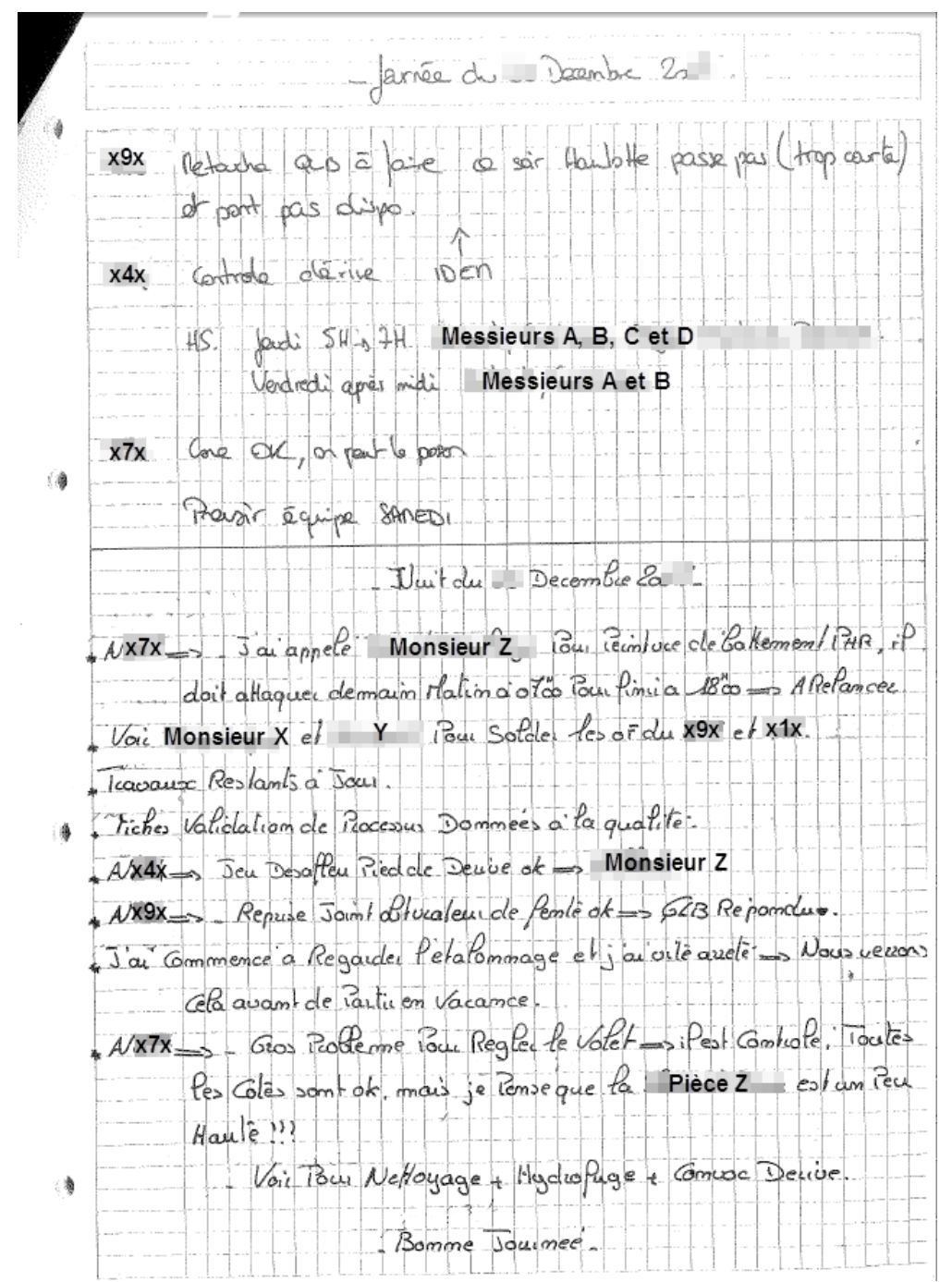

Figure 1 :" xtrait du cahier de liaison des chefs d'équipe

Figure 1: Extract from supervisor shift log

\section{3.- Méthodologie}

\section{1.- Le recueil de données}

Le recueil de données fut réalisé lors de 4 blocs de 2 jours d'observation répartis sur une période de 3 mois, et positionnés de façon identique dans le cycle d'assemblage de la station. Chaque bloc couvre 4 vacations successives de travail (Jour-Soir-Jour-Soir), soit 2 jours de fonctionnement de la station (cf. Figure 2). Deux observateurs ont été nécessaires pour suivre à tour de rôle chacun des chefs d'équipe : le premier affecté au chef d'équipe de jour et le deuxième à celui de soir. Au total cela représente 16 vacations ( 8 de jour et 8 de soir) donc 8 relèves avec rencontre et 8 relèves sans rencontre. 


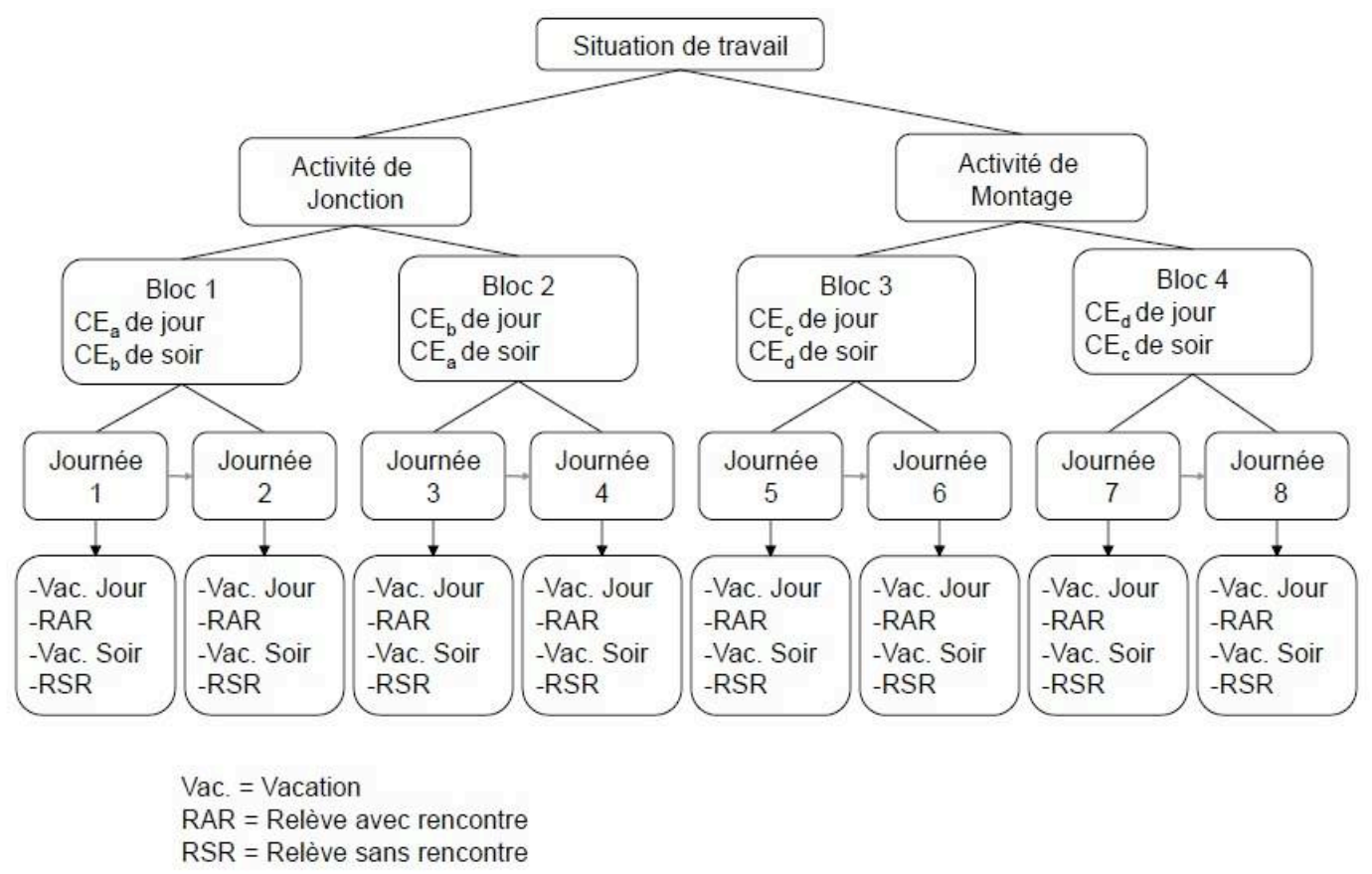

Figure 2 : Plan d'observation

Figure 2: Observation plan

Le contenu des cahiers de liaison des chefs d'équipe correspondant à ces 4 blocs d'observation a été conservé et analysé au regard du déroulement des vacations. Le déroulement des vacations a été recueilli en utilisant, de manière synchronisée, un enregistreur d'évènements et un carnet de bord. La grille d'observation numérique servant à encoder les événements a été construite au moyen du logiciel Actopalm ${ }^{\circledR}$ afin de réaliser une exploitation des données sous Actogram-Kronos (Kerguelen, 1991). Ce dernier a permis, lors du recueil, un encodage descriptif horodaté (lieux, documents et objets utilisés) et un encodage interprétatif a posteriori (Delgoulet, Kerguelen, \& Barthe, 2000). Les tâches poursuivies par les chefs d'équipe tout au long de la vacation, dont la tâche de transmissions écrites, et les éléments de contexte qui leur donnent sens ont été relevés et notés sur le carnet de bord. " n complément, les activités verbales orales du binôme ont été enregistrées lors des relèves avec rencontre.

\section{2.- Le traitement des données}

\subsection{1.- Les topics traités}

L'activité de travail des chefs d'équipe a été spécifiée selon l'objet du travail qu'ils traitaient dans leurs actions, leurs communications ou leurs rédactions tout au long des vacations.

Ainsi le processus de travail observé a été subdivisé, codé et analysé en termes de " topics » (terme repris de la linguistique) sur la base de différents types de données: pour les vacations, le carnet de bord des observateurs; pour les relèves sans rencontre, les transmissions rédigées sur le cahier de liaison; pour les relèves avec rencontre, les échanges oraux et les transmissions rédigés sur le cahier de liaison.

Un «topic »a été défini comme l'enchaînement des actions et communications relatives à un même objet du travail. L'objet du travail peut-être ponctuel (incident, modification de l'organisation) ou récurrent (réunion de service, suivi de l'avancement des opérations sur une zone de travail), nominal (déroulement habituel des opérations prescrites sur une zone de travail) ou dysfonctionnel (traitement d'un écart par rapport à la mise en œuvre d'une gamme de travail, à la mise en sécurité d'une zone de travail). 


\subsection{2.- Codage direct des topics : la finalité de l'évocation du topic}

Pour chaque topic transmis (par écrit et/ou à l'oral), la finalité générale de l'évocation du topic lors de la relève de poste a été identifiée. Cette finalité d'évocation d'un topic pouvait être de deux ordres (pour connaissance vs pour action) :

- Pour simplement porter à la connaissance de son homologue un état de fait : «toutes feuilles qualif manquantes faites »; «la benne est commandée ».

— Pour demander à l'homologue d'amorcer ou poursuivre une action sur un topic donné : « Relancer T"* A pour AM 27xyz18 (Ferrure sous voilure) »; « voir avec Maryse pour la perme de $[\ldots] »$.

\subsection{3.- La séquence de transmission et les actions de rédaction}

Les transmissions rédigées dans le cahier de liaison ont été découpées en séquences de transmission en suivant l'ordre chronologique de lecture (parfois différent de l'ordre de rédaction): dès que le topic change alors la séquence change. Ainsi une séquence écrite correspond au paragraphe relatif à un topic que l'opérateur montant lira.

De plus, l'opérateur descendant peut rédiger une même séquence en plusieurs fois. " $\mathrm{n}$ effet, il peut être interrompu par une tierce personne au cours de sa rédaction, il peut rédiger une consigne à un moment $t$ et la compléter ultérieurement. Pour pouvoir rendre compte de cette dynamique d'élaboration des transmissions écrites, nous avons rajouté sous ActogramKronos ${ }^{\circledR}$ une nouvelle catégorie d'observables : l'action de rédaction. L'action de rédaction représente l'instant $t$ auquel l'opérateur rédige une information relative à un topic donné. Pour une séquence de transmission écrite, il est possible d'observer plusieurs actions de rédaction différentes.

\subsection{4.- Codage indirect des topics à partir des séquences de rédaction}

Sur la base des actions de rédaction, chaque topic transmis par écrit a été catégorisé en fonction de :

- l'existence d'une continuité entre l'action et la rédaction (continu vs discontinu) : si l'opérateur ne traitait pas d'un autre topic entre le moment où l'information à transmettre était établie et le moment où cette information était mise par écrit dans le cahier de liaison, alors la première mise par écrit d'un topic a été considérée comme continue à l'action ;

— l'existence d'une modification ultérieure de la rédaction (modifié vs non modifié) : si la première action de rédaction était suivie d'autres actions de rédaction visant à compléter ou rectifier la première information mise par écrit, alors le topic était classé comme ayant fait l'objet d'une modification ultérieure.

\section{3.- Codage et analyses}

Les deux observateurs se relayaient à la même fréquence que les opérateurs étudiés. Dans un premier temps chacun, séparément, a codé l'ensemble des données recueillies. Dans un deuxième temps, ils ont mis en commun leurs codages. Les codages finaux sont le résultat d'une décision conjointe prise à l'issue d'une consultation entre les deux observateurs. Un des deux observateurs a une expérience significative de ce type de situation et du travail des chefs d'équipe. À partir de ce codage, les données quantitatives ont été traitées à l'aide de la statistique du Chi-2.

L'analyse qualitative sur la continuité de l'activité à travers des vacations successives est centrée sur le topic. Tout d'abord, les chronologies d'actions et de communications de chaque topic ont été isolées. " nsuite les topics transmis ont été retrouvés à partir du contenu des relèves de poste analysées. 


\section{4.- Résultats}

\section{1.- Des transmissions écrites systématiques pour les relèves sans rencontre}

Contrairement aux relèves sans rencontre, la rédaction d'écrits de transmission n'est pas systématique pour les relèves avec rencontre (cf. Tableau 1).

\begin{tabular}{|l|c|c|}
\hline \multicolumn{1}{|c|}{ Transmissions écrites } & $\begin{array}{c}\text { Relève } \\
\text { Sans Rencontre }\end{array}$ & $\begin{array}{c}\text { Relève } \\
\text { Avec Rencontre }\end{array}$ \\
\hline $\begin{array}{l}\mathrm{Nb} \text { de relèves de poste concernées / Nb de } \\
\text { relèves étudiées }\end{array}$ & $8 / 8$ & $4 / 8$ \\
\hline $\mathrm{Nb}$ cumulé de topics transmis & 78 & 25 \\
\hline $\begin{array}{l}\text { Moyenne de topics transmis par relève de } \\
\text { poste concernée }\end{array}$ & $10(\mathrm{SD}=2.0)$ & $6(\mathrm{SD}=5.3)$ \\
\hline
\end{tabular}

Tableau 1 : Répartition des transmissions écrites par type de relève de poste (avec vs sans rencontre)

Table 1: Distribution of topics transmitted in writing, by shift-changeover type (with or without meeting)

Les premiers résultats présentés concerneront la rédaction de transmissions écrites pour les relèves sans rencontre.

\section{2.- Une activité de rédaction tout au long de la vacation}

\subsection{1.- Des actions de rédaction pour les relèves sans rencontre tout au long de la vacation}

C'est entre $00 \mathrm{~h}$ et $1 \mathrm{~h}$, soit au moment de la plage flexible de départ, que la proportion horaire d'actions de rédaction ( $33 \%$ ) est la plus forte (cf. Figure 3 ). Toutefois les deux tiers des actions de rédaction de la période de $23 \mathrm{~h}-00 \mathrm{~h}$ ont été réalisées après $23 \mathrm{~h} 30$, soit dans la dernière demi-heure de présence des agents de fabrication sur la station de travail. C'est d'ailleurs dans cette dernière demi-heure que les chefs d'équipe réalisent une dernière ronde sur la station.

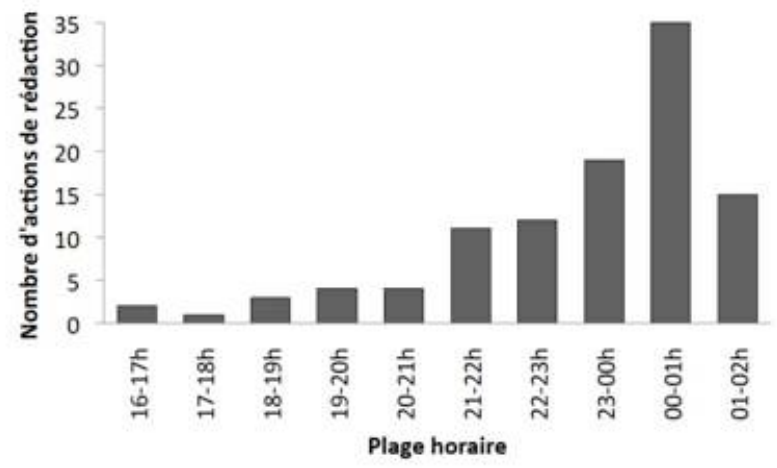

Figure 3 : Distribution horaire des actions de rédaction pendant les vacations de soir $(\mathrm{N}=106)$

Note : La pause repas de la vacation de soir intervient entre $19 \mathrm{~h} 30$ et $20 \mathrm{~h} 30$

Figure 3: Hourly distribution of writing tasks during evening shifts $(N=106)$ Note: The evening shift's meal break occurs between 7:30 and 8:30 p.m. 


\subsection{2.- Trouver le temps d'écrire au risque de devoir modifier une information transmise}

Les actions de rédaction de transmissions ne sont pas systématiquement continues de l'action réalisée, elles peuvent être différées dans le temps. Ainsi pour $67 \%$ des 78 topics transmis par écrit lors des relèves sans rencontre, la première action de rédaction n'a pas été réalisée dans la continuité de l'action : le chef d'équipe a traité un ou plusieurs autres topics avant de rédiger une transmission sur un topic en particulier.

De plus, l'insertion des actions de transmission dans le cours de l'activité de travail implique parfois de modifier ultérieurement une transmission préalablement rédigée c'est-à-dire de compléter une information (ex : rajouter le numéro d'un procès verbal) ou de corriger une partie d'une information (ex. : barrer "Cadre 38 Montage rail de soute. Il manque un sabot au cadre 37-4" et rajouter "SABOT retrouvé").

Ainsi pour $31 \%$ des topics (11 topics sur 35$)$ qui ont fait l'objet d'une première action de rédaction avant la fin de la vacation (avant 23h30), l'information transmise a dû ensuite être modifiée par le chef d'équipe, contre $9 \%$ des topics (4 topics sur 43) mis pour la première fois par écrit durant la période de fin de poste (après 23h30) (cf. Figure $4 ; \chi^{2}=4,740 ; \mathrm{ddl}=$ $1 ; \mathrm{p}=0,029)$.

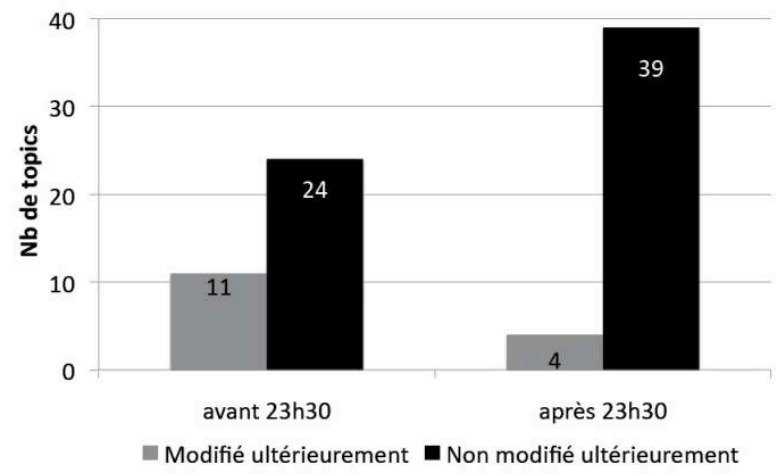

Figure 4 : Répartition des topics mis par écrit avant et après $23 \mathrm{~h} 30$ en fonction de l'existence d'une modification ultérieure de la première information rédigée

Figure 4: Distribution of topics written down before and after 11:30 p.m., according to whether the initial information in the written handover was subsequently modified

L'indisponibilité immédiate du chef d'équipe pour écrire une transmission tout de suite après l'action et la modification de transmissions déjà rédigées sont illustrées dans 1"' xtrait 1, qui présente un enchaînement résumé d'actions et de communications relatives à un même topic. 


\begin{tabular}{|c|c|c|}
\hline 1 & & $\begin{array}{l}\text { Soir 1: } \mathrm{CE}_{\text {soir avait rédigé une transmission pour son homologue de jour à }} \\
\text { propos du prêt d'un "pistolet » (alias visseuse) à un agent de fabrication } \\
\left(\mathrm{AF}_{1}\right) \text { d'une autre station: } \\
\text { Transmission écrite (Soir 1): P35 AC-Prêt de } 2 \text { pistolets automatiques }\end{array}$ \\
\hline 5 & $19 \mathrm{~h} 22$ & $\begin{array}{l}\text { diamètre 4,8 }(\mathrm{Mr} \text { BARRI). } \\
\text { Soir } 2 \text { : Un deuxième agent de fabrication }\left(\mathrm{AF}_{2}\right) \text { de l'autre station demande } \\
\text { un pistolet automatique au } \mathrm{CE}_{\text {soir. }} \mathrm{CE}_{\text {soir }} \text { dit à } \mathrm{AF}_{2} \text { qu'il en a déjà prêté deux } \\
\text { la veille au soir à } \mathrm{AF}_{1} \text { et qu'il les suppose non restitués puisque } \mathrm{CE}_{\text {jour ne lui }} \\
\text { a rien transmis à ce sujet. }\end{array}$ \\
\hline \multirow[t]{2}{*}{10} & & 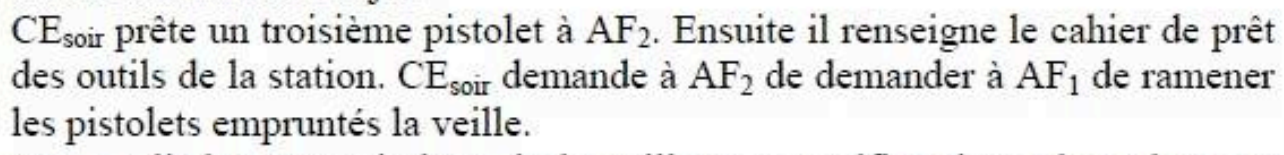 \\
\hline & $19 \mathrm{~h} 26$ & $\begin{array}{l}\mathrm{CE}_{\text {soir relit les transmissions de la veille pour vérifier si son homologue a }} \\
\text { noté un évènement nouveau sur ce topic. }\end{array}$ \\
\hline 15 & & $\begin{array}{l}\mathrm{CE}_{\text {soir rédige une nouvelle transmission : }} \\
\text { Transmission écrite (Soir } 2 \text { ): } P 35 \mathrm{~A}-\text { Prêt de } 1 \text { pistolet automatique à Mr } \\
\text { DELVE cela fait } 3 \text { pistolets en } 2 \text { jours. Attention au retour en état de } \\
\text { fonctionnement. }\end{array}$ \\
\hline \multirow[t]{2}{*}{20} & $21 \mathrm{~h} 00$ & $\begin{array}{l}\mathrm{CE}_{\text {soir }} \text { est sur la station au niveau du cadre de jonction arrière. } \mathrm{AF}_{2} \text {, qui est } \\
\text { présent, dit à } \mathrm{CE}_{\text {soir }} \text { qu'il a ramené le troisième pistolet automatique emprunté } \\
\text { plus tôt. }\end{array}$ \\
\hline & $22 \mathrm{~h} 38$ & 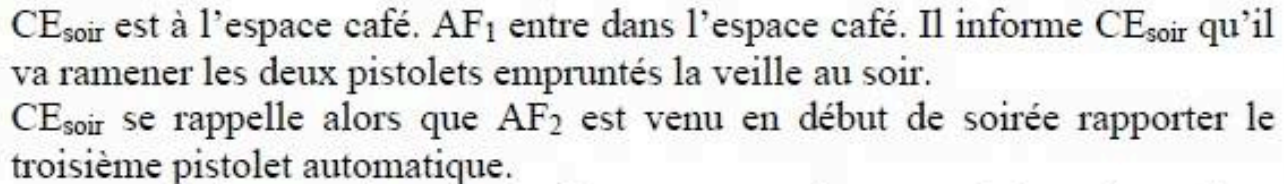 \\
\hline 25 & $22 \mathrm{~h} 49$ & $\begin{array}{l}\mathrm{CE}_{\text {soir est assis à son bureau. Il rédige une nouvelle transmission, située deux }} \\
\text { transmissions en dessous de celle rédigée précédemment sur ce même topic : } \\
\text { Transmission écrite (Soir } 2 \text { ): } P 35 \text { A C - Mr Delve a ramené } 1 \text { pistolet } \\
\text { auto. }\end{array}$ \\
\hline
\end{tabular}

" xtrait $1:$ : nchaînement d'actions et de communications (résumées) d'un chef d'équipe de soir sur un topic qu'il transmettra par écrit à la relève sans rencontre suivante

Excerpt 1: Series of actions and communications (summarised) by an evening shift supervisor concerning a topic he will transmit in writing to the next shift changeover without meeting

\section{3.- Utilisation des écrits pour se rappeler les actions à poursuivre tout au long de la vacation}

\subsection{1.- Transmettre pour informer ou pour agir}

Selon le type de relève de poste, les objectifs poursuivis lors de la rédaction d'une transmission écrite sont significativement différents $\left(\chi^{2}=3,858\right.$; ddl $\left.=1 ; \mathrm{p}=0,049\right)$ (cf. Figure 5): les transmissions écrites des relèves sans rencontre sont majoritairement des demandes d'action adressées au chef d'équipe montant de jour (64\% des topics transmis de relève sans rencontre) alors que les transmissions écrites des relèves avec rencontre visent en majorité à informer le chef d'équipe montant de soir sur un état de fait (68\% des topics transmis de relève avec rencontre). 


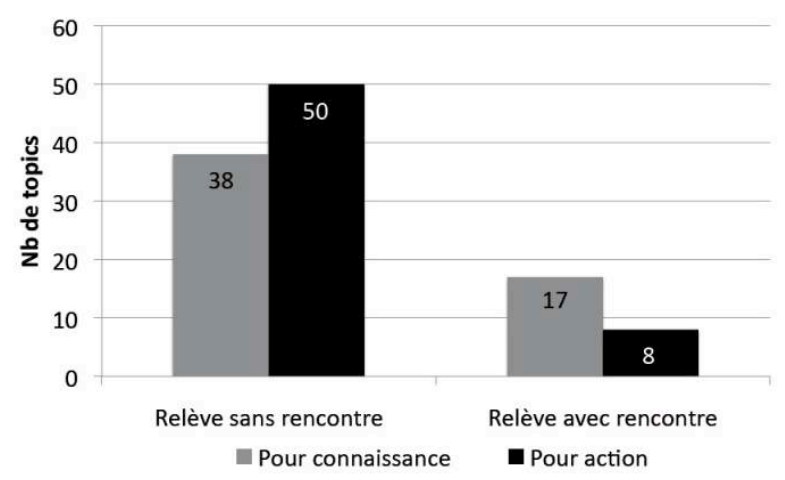

Figure 5 : Distribution des topics transmis par écrit en fonction du type de relève de poste (avec vs sans rencontre) et de l'objectif poursuivi par la rédaction de la transmission (pour connaissance vs pour action).

Figure 5: Distribution of topics transmitted in writing, by shift-changeover type (with or without meeting) and by the objective of the written handover (to inform vs. to take action).

\subsection{2.- Utilisation des écrits de la veille par le chef d'équipe montant}

Les demandes d'action adressées au chef d'équipe montant étant plutôt spécifiques des relèves sans rencontre (cf. Figure 5), la suite de l'analyse s'appuie sur l'activité du chef d'équipe de jour en lien avec le cahier de liaison.

Au cours de 6 vacations de jour (sur les 8 étudiées), les chefs d'équipe ont utilisé le cahier de liaison à plusieurs reprises. L'analyse de ces usages montre le rôle du cahier de liaison dans la mise en œuvre de certaines actions et dans le traçage des actions menées. Ces deux aspects sont illustrés à travers la schématisation d'une des 6 vacations identifiées pour cette analyse (cf. Figure 6). " lle présente le contenu des écrits précédant la vacation de jour et le contenu des activités du chef d'équipe montant de jour en rapport avec les topics (B, F, G, H et I) transmis dans la relève sans rencontre.

La Figure 6 montre que le chef d'équipe de jour utilise le cahier de liaison tout au long de sa vacation, régulièrement de $7 \mathrm{~h} 14$ à $15 \mathrm{~h} 08$, et ce de différentes manières.

La première stratégie est de surligner les transmissions rédigées par l'homologue lors de la relève sans rencontre, une fois que l'action demandée est réalisée : à $7 \mathrm{~h} 22$, à $8 \mathrm{~h} 18$, à $8 \mathrm{~h} 49$, à $10 \mathrm{~h} 04$.

La deuxième stratégie est d'annoter des transmissions rédigées par l'homologue lors de la relève sans rencontre pour apporter des précisions sur l'état de l'action réalisée et les informations obtenues. Ainsi il rajoute des «Ok soldé » (cf. 1. 18), «Aujourd'hui » (cf. 1. 24), « Ok» (cf. 1. 32) au bout de transmissions de demande d'action rédigées la veille par son homologue.

La troisième stratégie est de rédiger de «nouvelles » transmissions dans une nouvelle partie du cahier de liaison (en dessous de celles de la relève sans rencontre) quand un aléa important a empêché le bon déroulement de ce qui était attendu ou bien pour informer sur un nouveau topic (cf. 1.39 à 41).

Ici le cahier de liaison est un point focal qui permet au chef d'équipe de jour (1) de se réactiver sur les actions que son homologue de soir lui demande de poursuivre et (2) de faire en retour un état des résultats obtenus à ces demandes d'action, et surtout (3) de les tracer en combinant plusieurs stratégies pour compléter le cahier de liaison. 


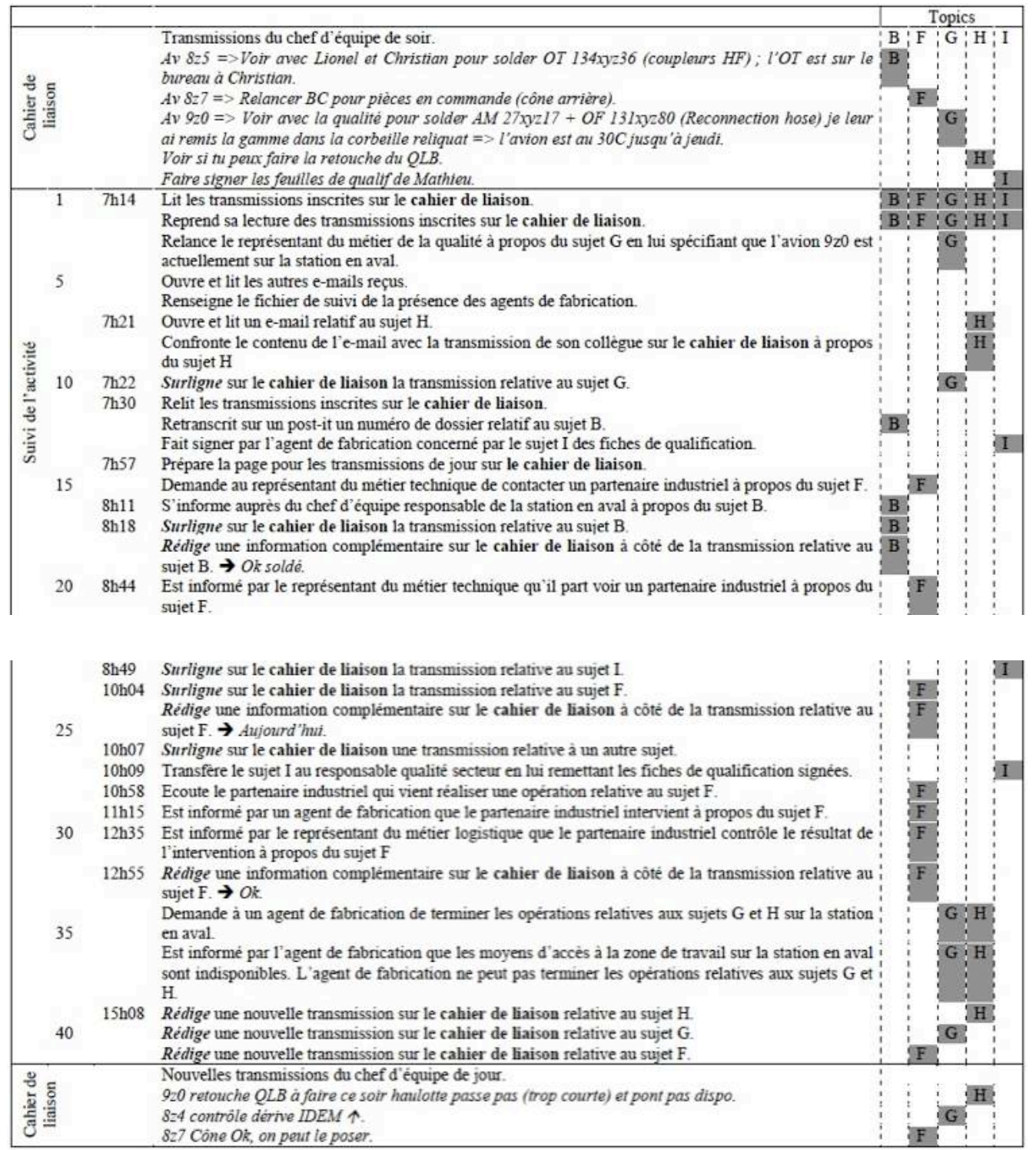

Figure 6 : Usage fait du cahier de liaison et des transmissions qui y sont rédigées pendant la vacation de jour

Note : Le premier cadre présente les transmissions rédigées par le chef d'équipe de soir sur le cahier de liaison (en italique). Le deuxième cadre présente l'enchaînement horodaté des actions du chef d'équipe de jour au cours de sa vacation. Le troisième cadre présente les nouvelles transmissions rédigées par le chef d'équipe de jour sur le cahier de liaison. Dans la colonne de droite et en gris sont identifiés les différents topics évoqués (B, F, G, H et I) dans les transmissions écrites, et traités par le chef d'équipe au cours de sa vacation.

Figure 6: Use of the shift log and handovers written down throughout the day shift

Note: The upper box presents the evening shift supervisor's handovers written down on the shift log (in italics). The middle box shows the time-labelled series of actions carried out by the day-shift supervisor during his shift. The bottom box presents the day-shift supervisor's new handovers written down on the shift $\log$. The different topics $(B, F, G, H$, and I) mentioned and processed in the written handovers by the shift supervisor during his shift are shown in grey on the right.

La Figure 6 montre que le chef d'équipe de jour utilise le cahier de liaison tout au long de sa vacation, régulièrement de $7 \mathrm{~h} 14$ à $15 \mathrm{~h} 08$, et ce de différentes manières. 
La première stratégie est de surligner les transmissions rédigées par l'homologue lors de la relève sans rencontre, une fois que l'action demandée est réalisée : à $7 \mathrm{~h} 22$, à $8 \mathrm{~h} 18$, à $8 \mathrm{~h} 49$, à $10 \mathrm{~h} 04$.

La deuxième stratégie est d'annoter des transmissions rédigées par l'homologue lors de la relève sans rencontre pour apporter des précisions sur l'état de l'action réalisée et les informations obtenues. Ainsi il rajoute des «Ok soldé » (cf. 1. 18), «Aujourd'hui » (cf. 1. 24), « Ok» (cf. 1. 32) au bout de transmissions de demande d'action rédigées la veille par son homologue.

La troisième stratégie est de rédiger de «nouvelles» transmissions dans une nouvelle partie du cahier de liaison (en dessous de celles de la relève sans rencontre) quand un aléa important a empêché le bon déroulement de ce qui était attendu ou bien pour informer sur un nouveau topic (cf. 1.39 à 41).

Ici le cahier de liaison est un point focal qui permet au chef d'équipe de jour (1) de se réactiver sur les actions que son homologue de soir lui demande de poursuivre et (2) de faire en retour un état des résultats obtenus à ces demandes d'action, et surtout (3) de les tracer en combinant plusieurs stratégies pour compléter le cahier de liaison.

\section{4.- Les écrits : un outil pour les échanges oraux pendant la relève avec rencontre}

\subsection{1.- Utilisation des écrits de la relève sans rencontre lors de la rencontre suivante}

Les transmissions écrites de la dernière relève sans rencontre évoquent 11 topics différents (de $\mathrm{A}$ à $\mathrm{K}$ ) évoqués à travers 12 séquences de transmission (le topic $\mathrm{G}$ étant évoqué dans deux paragraphes distincts) (cf. Figure 7). Parmi ces 11 topics, 4 topics sont transmis à titre d'information, et 7 afin que le montant (de jour) poursuive une action.

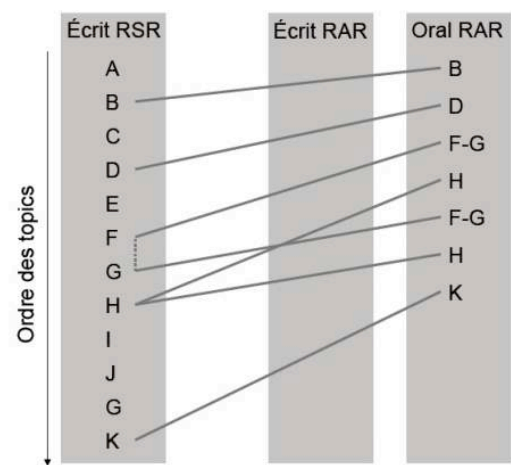

Figure 7 : Schématisation de l'ordre de présentation, sur le cahier de liaison, des topics rédigés lors de la relève sans rencontre (RSR) précédente et de l'ordre

d'évocation à l'oral des topics lors de la relève avec rencontre (RAR)

Note : Les traits symbolisent que l'évocation des topics s'appuie sur le contenu du cahier de liaison

Figure 7: Schematic drawing of the order of topics written down in the shift log during the previous without-meeting changeover (RSR), and order of topics mentioned orally during the with-meeting changeover (RAR) Note The lines connect comments from the shift log's content

Pendant la relève avec rencontre, l'ordre d'évocation de ces topics suit l'ordre de rédaction des transmissions de la dernière relève sans rencontre. Les chefs d'équipe, et principalement le descendant, passent préférentiellement en revue les topics pour lesquels le montant (de soir) avait formulé des attentes au moment de la rédaction des transmissions la veille. 


\subsection{2.- Utilisation des transmissions écrites de la relève avec rencontre lors de la rencontre}

Les transmissions rédigées pour la relève sans rencontre et pour la relève avec rencontre étaient rédigées sur deux pages en face à face, de telle sorte qu'à l'ouverture du cahier de liaison elles s'offraient simultanément à la vue du lecteur.

Les transmissions écrites de la relève sans rencontre évoquent 9 topics différents (de A à I). Parmi les 9 topics, 2 topics sont transmis à titre d'information, et 7 dans le but que le montant (de jour) poursuive une action (cf. Figure 8).

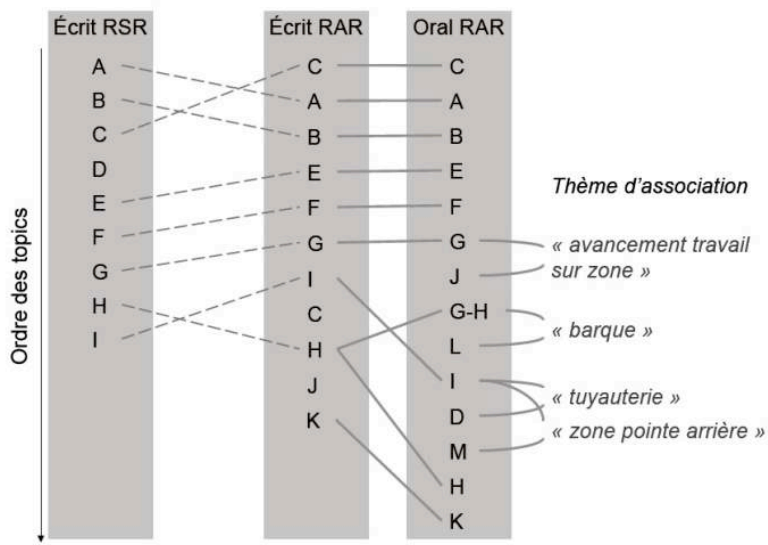

Figure 8 : Schématisation de l'ordre de présentation, sur le cahier de liaison, des topics rédigés lors de la relève sans rencontre (RSR) précédente et pour la relève avec rencontre (RAR) en cours, et de l'ordre d'évocation à l'oral des topics lors de la relève avec rencontre

Note : Les traits en pointillés entre « écrit RSR » et « écrit RAR » symbolisent la continuité entre relève sans rencontre et relève avec rencontre. Les traits pleins symbolisent que l'évocation des topics s'appuie sur le contenu du cahier de liaison

Figure 8: Schematic drawing of the order of topics written in the shift logs, for the previous without-meeting changeover (RSR) and for the current withmeeting changeover $(R A R)$, and the order of the topics mentioned orally during the with-meeting changeover

Note: The dotted lines between "écrit RSR" and "écrit RAR" symbolise the continuity between the without-meeting changeover and the with-meeting changeover. The solid lines connect comments from the shift log's content

Les transmissions écrites de la relève avec rencontre (rédigées par le chef d'équipe de jour descendant) évoquent 10 topics différents à travers 11 séquences de transmission (le topic $\mathrm{C}$ est évoqué dans deux paragraphes différents). Parmi les 10 topics, 8 topics avaient déjà été évoqués dans les transmissions précédentes de la relève sans rencontre (topics A, B, C, " , F, $\mathrm{G}, \mathrm{H}$ et I). De manière générale, les topics sont transmis à des fins d'information.

L'analyse montre ici aussi que la trame des transmissions écrites sert de fil conducteur aux échanges oraux de la relève avec rencontre suivante : du topic $\mathrm{C}$ au topic $\mathrm{G}$.

à partir du topic $\mathrm{G}$, les échanges oraux s'écartent de l'ordre de ce qui est écrit pour ainsi évoquer des topics supplémentaires par association d'idées :

- l'évocation de l'avancement d'une opération qui a posé problème sur une zone de travail du train d'atterrissage gauche (topic G) entraine l'évocation de l'avancement du travail sur les autres zones de travail de l'avion : le topic $\mathrm{J}$ relatif aux glissières montées sur les voilures de l'avion ("Le client glissière est passé ») et ensuite simultanément les topics G et $\mathrm{H}$ pour planifier les opérations à réaliser à droite et à gauche ("ce soir faut que je fasse les barques») ; 
- l'évocation des opérations de montage des barques sur l'avion en position sur la station (topics $\mathrm{G}$ et $\mathrm{H}$ ) induit l'évocation d'une opération en reliquat à réaliser sur les barques de l'avion positionné sur la station en aval (topic L : «il faut les finir là-bas! ») ;

- l'évocation d'un problème sur des tuyauteries de la pointe arrière (topic I) induit l'évocation par le montant d'un topic problématique relatif à d'autres tuyauteries (topic D : «Et les trucs... Il l'avait bien fait, il y avait les bons numéros, ils étaient bons?»), ainsi que l'évocation de la réalisation d'une opération de réglage en pointe arrière n'ayant pas fait l'objet de transmissions écrites (topic $\mathrm{M}$ : «Le tream, on n'y a pas touché bien sûr »).

Toutefois, l'écrit reste un point d'ancrage qui permet, au descendant comme au montant, de reprendre le fil après s'être écarté de la trame écrite :

— quand le chef d'équipe descendant évoque le topic I ("L'arrivée tuyauterie, demain matin...»);

— quand le chef d'équipe descendant revient sur le travail à faire par l'évocation du topic $\mathrm{H}$ ( Donc concrètement à droite, il reste »).

\section{5.- Éléments de réflexion pour l'aménagement des relèves de poste de l'encadrement de proximité}

Les premiers résultats montrent que la rédaction des transmissions par les chefs d'équipe est systématique à l'approche d'une relève de poste sans rencontre et s'effectue tout au long de la vacation. Toutefois les nombreuses interruptions inhérentes aux tâches et aux missions du métier d'encadrement de proximité qui imposent à l'opérateur de s'engager dans un autre sujet, l'éloignement de son bureau où se trouve(nt) bien souvent le(s) $\operatorname{support}(\mathrm{s})$ de transmission conduisent l'encadrant de proximité à reporter dans le temps la rédaction d'une transmission qu'il juge utile, au risque de ne plus s'en souvenir quand les conditions seront réunies pour réaliser cette tâche de rédaction. Ainsi l'indisponibilité de l'encadrement de proximité, inhérente à leur activité de travail, pose la question de la mémorisation des informations et de leur recouvrement à un moment où il sera dans des conditions propices pour une rédaction de relève de poste. L'enjeu est alors de pouvoir intégrer dans son activité des «moments d'écriture » au pluriel, comme l'a souligné Delcambre (1993) à propos des éducateurs spécialisés.

Le moment d'écriture alimente aussi la question de la validité des informations transmises. " n effet nos résultats ont montré que pour une information mise par écrit bien avant la fin de la vacation, le risque est plus grand qu'elle soit modifiée ultérieurement dans la vacation, parce qu'une nouvelle information ou un nouvel événement remet en question la première information rédigée. L'évolution de la transmission au cours de la vacation, du fait du caractère dynamique de la situation (Cellier, De Keyser, \& Valot, 1996), engendre alors des ratures ou des corrections de ce qui a été écrit plus tôt.

Un parallèle est à faire avec les pratiques d'écriture non visibles, non reconnues et ne faisant pas partie de la description officielle des postes et des qualifications. Ce sont les «petits cahiers ou des petits bouts de papier, enfouis dans les poches des combinaisons et des blouses et sur lesquels on consigne un ensemble de remarques, d'observations, de calculs à propos du fonctionnement des machines, des processus de fabrication» (Boutet, 2008, p. 71). Il s'agirait alors d'écritures ébauchoirs dont l'objectif est d'exprimer, fixer et former sa pensée (Doquet-Lacoste, 2009) dans l'instant afin de faciliter ultérieurement le recouvrement des informations lors de moments propices à la rédaction de transmissions sur un support de transmission par exemple.

Nos résultats ont montré que les transmissions écrites permettaient de tracer sa propre activité, de guider celle de son homologue et de guider les transmissions orales lors de la 
rencontre. Plus généralement le support de transmission est un outil pour fixer les informations nécessaires à la coordination et à la continuité. Son usage n'est pas limité au seul moment du changement d'équipe, mais s'inscrit dans l'activité continue de l'encadrement de proximité. Le format du support de transmission (un simple cahier A4) permet une rédaction libre où les transmissions sont inscrites les unes à la suite des autres. C'est un flot continu qui alterne des transmissions pour informer et des transmissions qui demande d'agir ("AM à faire », "relancer », etc.). Nos résultats ont montré que le montant complète les demandes d'action de celui qui n'est plus là pour rendre compte de façon explicite (annotation) ou implicite (surlignage) de l'état de l'action qu'il devait poursuivre ("ok soldé », " aujourd'hui » dans les cas où c'est explicite). Ainsi si les transmissions de relève avec rencontre sont plus informationnelles, c'est parce que ce sont souvent des réponses aux demandes formulées dans les transmissions écrites de relève sans rencontre.

À travers les transmissions écrites, l'encadrement de proximité organise les tâches qui seront réalisées par les collectifs de travail dans lesquels ils sont impliqués. Cette coordination alterne dans le temps entre le niveau formel et le niveau informel (Leplat \& Savoyant, 1983 ; Maggi, 1996 ; Rabardel, Rogalski, \& Béguin, 1996). Quand le descendant organise et prescrit les actions à réaliser par son homologue montant, on peut considérer qu'une coordination formelle s'effectue à l'approche des relèves. Les transmissions permettent de guider l'activité collective. Cette coordination formelle active en priorité le collectif temporellement distribué. La coordination informelle, contextuelle à l'action et menée par la personne agissante, intervient quand l'opérateur montant réalise au fil de sa vacation les demandes d'action de son homologue descendant. Cette coordination informelle entraîne alors l'activation des collectifs immédiats et périphériques. L'écriture de ce qui est à faire et en retour de ce qui a été fait ou pas, supporte et trace la coordination de l'activité collective.

L'analyse présentée s'est centrée sur le principal support de transmission des chefs d'équipe. Les transmissions de l'encadrement de proximité passaient par d'autres supports (comme l'e-mail pour transférer des informations plus confidentielles) et s'appuyaient aussi sur d'autres objets de la situation de travail, que nous n'avons pas relevés systématiquement, qu'ils instrumentalisaient pour se souvenir d'une information à transmettre et plus généralement pour régler leur coordination temporellement distribuée.

Ces objets « instrumentalisés » (Rabardel, 1995) sont utilisés comme un moyen d'action par l'opérateur. Pour l'activité de relève de poste, cela correspond à l'ensemble des objets du travail (de la gamme de travail à une pièce défectueuse, etc.) qui seront détournés pour transmettre les informations d'une vacation à une autre dans la lignée de la cognition distribuée (Hutchins, 1995). Comme les contrôleurs aériens qui communiquent en modifiant des éléments du contexte (exemple de la disposition des strips, Cox, Sharples, Stedmon, \& Wilson, 2007), les opérateurs postés organisent spatialement les documents et les objets porteurs d'informations. Le descendant les instrumentalise pour les rendre plus visibles aux yeux du montant. Le pré-requis pour le montant est qu'il détienne les connaissances qui lui permettront d'interpréter les implicites : par exemple si une gamme de travail est posée sur le bureau avec un post-it collé indiquant un numéro de procès-verbal, cela signifie qu'il y a eu un problème sur cette gamme et qu'il est en cours de traitement. Lors des relèves sans rencontre, ces pratiques participent à guider la prise d'informations du montant vers des points importants à traiter ou à connaître en s'affranchissant du coût d'une rédaction explicite. Lors des relèves avec rencontre, c'est aussi un moyen pour le descendant de se rappeler les points à discuter avec son collègue. Dans ce cas, les différents objets de la situation constituent des éléments évocateurs qui faciliteront le rappel des informations, et qu'ils sauront exploiter pour ajuster mutuellement leurs représentations de la situation. Une perspective à notre étude serait alors d'étudier de manière plus systématique l'instrumentalisation des objets de la situation dans le cadre des relèves de poste.

L'ensemble des résultats présentés questionne le modèle de la relève de poste qui envisage la 
relève de poste comme un moment du travail posté, positionné sur la fin de la vacation qui s'achève et sur le début de la vacation qui commence. Certains auteurs ont déjà souligné des distorsions relatives à ce déroulement. Ils ont par exemple observé des infirmières rédiger leurs transmissions après la rencontre orale (Boucheix \& Coiron, 2008 ; Hawley, Stilwell, Robinson, \& Bond, 1995, cités par Hardey, Payne, \& Coleman, 2000) alors que l'activité de rédaction est préférentiellement réalisée et préconisée pendant la phase de préparation de la relève de poste, sous-entendu avant la rencontre.

Au-delà de l'entre-laçage des phases (rédaction pendant ou après la rencontre, prise de poste avant la rencontre si le descendant est retenu par une réunion), c'est la notion de moment qui est remise en question. " n effet hormis la rencontre qui est facilement identifiable pour tout intervenant extérieur qui souhaite organiser le travail posté à travers les relèves de poste, il est beaucoup plus difficile de dire quand commence par exemple la préparation de la relève de poste. Si un chef d'équipe ré-ordonne des documents ou objets posés sur son bureau, estce un simple rangement ou bien une action de préparation des informations à transmettre ? Il est donc réducteur d'aborder la relève de poste de l'encadrement de proximité, ou de tout autre opérateur qui doit gérer de la continuité et de nombreuses interfaces, comme un moment à organiser. Ce serait la même méprise que de réduire la relève de poste à un outil qui permettrait la suppression du chevauchement d'équipes par une organisation lors de l'aménagement du temps de travail.

" n effet pour l'encadrement de proximité, dont il est question ici, la finalité de son activité n'est pas la relève de poste, mais bien la continuité des activités dans le temps. " $t$ à ce titre, la préparation du changement d'équipe (rédiger des transmissions, instrumentaliser des objets de la situation) se gère et doit pouvoir se réaliser au fil de l'eau. Toute instrumentalisation des relèves de poste doit tenir compte des caractéristiques de l'activité des opérateurs postés concernés.

Nous n'avons pas mesuré l'effet des relèves de poste sur la performance et la fiabilité. Obtenir des mesures fiables de la performance dans le but de tester les bénéfices des relèves de poste reste difficile. Une des raisons est relative à la longueur des cycles de production qui dépassent la durée des vacations, avec des cycles d'assemblage final longs et des délais de réponse longs pour des problèmes logistiques ou techniques. Ces caractéristiques compliquent l'attribution d'une cause d'un problème donné aux relèves de poste. À cela se rajoutent les régulations réalisées par les différents opérateurs qui composent les collectifs de travail (immédiat, périphérique et temporellement distribués). Ces régulations qui visent à rattraper ici les éventuelles lacunes de transmissions, camouflent de fait une partie de la réalité aux autres membres des collectifs dont l'encadrement de proximité. Aubert (2009) a montré comment un indicateur de performance utilisé par des managers pour prendre des décisions peut masquer toute une activité dimensionnante de rattrapage de défauts réalisée par des agents de fabrication. Pour convaincre les organisations de l'enjeu d'aménager les relèves de poste, il est nécessaire de trouver des indicateurs de mesure de la performance et de la fiabilité des relèves de poste selon plusieurs axes : par exemple une auto-évaluation de la performance par l'encadrement de proximité après la vacation et à la fin du cycle de production, une évaluation de la charge mentale de l'encadrement de proximité, un recueil de la durée de réalisation de certaines opérations, etc.

Les résultats de la présente étude montrent que la relève de poste est un moment non circonscrit temporellement, et qu'à ce titre la tâche de transmission est une tâche transversale aux autres tâches de l'encadrement de proximité et qui participe à la coordination du travail à travers les vacations. Il en ressort deux principes majeurs pour l'optimisation des relèves de poste dont le pré-requis est de faire reconnaître par l'organisation que cette activité de relève de poste s'inscrit tout au long des postes. Si cette activité est reconnue par l'organisation comme telle, cela permettra dans un second temps de donner les moyens aux opérateurs de constituer des traces tout au long de leur activité. Ainsi le premier principe vise à favoriser la 
préparation de traces de l'activité de travail en permettant aux opérateurs de fixer les informations au cours de leur action. Le second principe est de structurer le support de transmission de relève de poste à partir d'indicateurs pertinents vis-à-vis de l'activité de travail qui permettraient d'évoquer, de repérer et de classer facilement les informations transmises et de suivre la continuité des actions.

\section{BIBLIOGRAPHI"}

Arora, V., Johnson, J., Lovinger, D., Humphrey, H.J., \& Meltzer, D.O. (2005). Communication failures in patient sign-out and suggestions for improvement: a critical incident analysis. Quality and Safety of Health Care, 14, 401-407.

Aubert, S. (1998). Connaissances incorporées et règles collectives : à la découverte des "savoirtravailler ensemble". Performances Humaines \& Techniques, 96, 30-37.

Aubert, S. (2009). Atelier « La pratique en pratique » Trois expériences d'ergonomes relatives à la performance. In Actes des Journées de Bordeaux (pp. 91-96).

Barthe, B. (2003). La visibilité de l'activité d'autrui, composante de la dimension collective du travail de soin. Travail et Emploi, 94, 51-58.

Barthe, B., \& Quéinnec, Y. (1999). Terminologies et perspectives d'analyse du travail collectif en ergonomie. L'Année Psychologique, 99(4), 663-686.

Bellies, L., \& Beauguil, L. (2008). L'accompagnement des projets de conception avec et sans objets intermédiaires : conséquence sur les coopérations entre acteurs de la conception. In P. Négroni \& Y. Haradji (" ds.), Ergonomie \& Conception. Concevoir pour l'activité humaine. 43ème Congrès de la S" LF, Ajaccio, 17-19 septembre (pp. 244-252). Ajaccio:" ditions ANACT.

Benchekroun, T.H. (2000). Les espaces de coopération proxémique. In T.H. Benchekroun \& A. Weill-Fassina (" ds.), Le travail collectif : Perspectives actuelles en ergonomie (pp. 35-54). Toulouse: Octarès.

Bisseret, A. (1970). Mémoire opérationnelle et structure de travail. Bulletin de Psychologie, 24(5-6), 280-294.

Boucheix, J.-M., \& Coiron, M. (2008). Analyse de l'activité de transmission écrite au cours des relèves de poste à l'hôpital : Évaluation ergonomique de l'usage d'un nouveau format d'écriture. Activités, 5(1), 79-102.

Bourdon, F., \& Weill-Fassina, A. (1994). Réseau et processus de coopération dans la gestion du trafic ferroviaire. Le Travail Humain, 57(3), 271-287.

Boutet, J. (2008). La vie verbale au travail : Des manufactures aux centres d'appels. Toulouse: Octarès.

Cahour, B., \& Falzon, P. (1991). Assistance à l'opérateur et modélisation de sa compétence. Intellectica, 2(12), 159-186.

Caroly, S. (2010). L'activité collective et la réélaboration des règles : des enjeux pour la santé au travail. Thèse d'Habilitation à Diriger une Recherche, Université de Bordeaux II, Bordeaux.

Caroly, S., \& Clot, Y. (2004). Du travail collectif au collectif de travail : développer des stratégies d'expérience. Forlmation Emploi, 88, 43-55.

Cellier, J.M., De Keyser, V., \& Valot, C. (1996). La gestion du temps dans les environnements dynamiques. Paris: PUF.

Cheung, D.S., Kelly, J.J., Beach, C., Berkeley, R.P., Bitterman, R.A., Broida, R.I., Dalsey, W.C., Farley, H.L., Fuller, D.C., Garvey, D.J., Klauer, K.M., McCullough, L.B., Patterson, “ .S., Pham, J.C., Phelan, M.P., Pines, J.M., Schenkel, S.M., Tomolo, A., Tubiak, T.W., Vozenilek, J.A., Wears, R.L., \& White, M.L. (2010). Improving handoffs in emergency department. Annals of Emergency Medicine, 55(2), 171-180.

Coleman, “ .A., \& Berenson, R.A. (2004). Lost in transition: Chanllenges and opportunities for improving the quality of transitional care. Annals of Internal Medicine, 140, 533-536.

Cook, R.I, Render, M., \& Woods, D.D. (2000). Gaps in the continuity of care and progress on patient safety. Education and Debate, 320, 791-794.

Cox, G., Sharples, S., Stedmon, A., \& Wilson, J. (2007). An observation tool to study air traffic control and flighdeck collaboration. Applied Ergonomics, 38(4), 425-435. 
Craik, F.I.M., \& Lockhart, R.S. (1972). Levels of processing: a framework for memory research. Journal of Verbal Learning and Verbal Behavior, 11, 671-684.

D'Argembeau, A., Van der Linden, M. (2006). Individual differences in the phenomenology of mental time travel: The effect of vivid visual imagery and emotion regulation stratégies. Consciousness \& cognition, 15(2), 342-350.

Davillerd, C., \& Grusenmeyer, C. (1993). La relève de poste : une période spécifique de travail collectif. In F. Six \& X. Vaxevanoglou (“ ds.), Les aspects collectifs du travail (pp. 81-88). Toulouse: Octarès.

Delcambre, P. (1993). Journal de bord et commis aux écritures. Empan, 11, 14-19.

Delcambre, P. (1997). Ecriture et communications de travail : pratiques d'écriture des éducateurs spécialisés. Villeneuve d'Ascq: Presses Universitaires du Septentrion.

Delgoulet, C., Kerguelen, A., \& Barthe, B. (2000). Vers une analyse intégrée des communications et des actions au travail : quelles modalités de leur mise en relation? 35ème Congrès de la SELF, Toulouse, France, Septembre.

Dieumegard, G., Saury, J., \& Durand, M. (2004). L'organisation de son propre travail : une étude du cours d'action de cadres de l'industrie. Le Travail Humain, 67(2), 157-179.

Doquet-Lacoste, C. (2009). “ crits intermédiaires, écritures intermittentes. Carnets, notes, bribes de science. Langage \& Société, 127, 7-22.

Giboin, A. (2004). La construction de référentiels communs dans le travail coopératif. In J.-M. Hoc \& F. Darses (“ ds.), Psychologie ergonomique : tendances actuelles (pp. 119-139). Paris: Presses Universitaires de France.

Goodwin, C., \& Goodwin, M.H. (1997). La coopération au travail dans un aéroport. Réseaux, 85, 129162.

Gramopadhye, A.K., Kelkar, K., \& Watson, J. (1999). Analysis of shift change in the aircraft maintenance environment: findings and recommendations. FAA Office of the Aviation Medicine.

Grosjean, M. (2005). L'awareness à l'épreuve des activités dans les centres de coordination. Activités, 2(1), 76-98. http://www.activites.org/v2n1/grosjean.pdf

Grosjean, M., \& Lacoste, M. (1999). Communication et intelligence collective : Le travail à l'hôpital. Paris: Presses Universitaires de France.

Grusenmeyer, C. (1991). La relève de poste : une phase critique du travail en équipes successives. Cahiers de Notes Documentaires, 144(3), 407-418.

Grusenmeyer, C. (1995). Interaction langagière et représentation mentale partagée : une étude de la relève de poste. Psychologie Française, 40(1), 47-59.

Hales, C.P. (1986). What do managers do? A critical review of the evidence. Journal of Management Studies, 23 (1), 88-115.

Hardey, M., Payne, S., \& Coleman, P. (2000). 'Scraps': hidden nursing information and its influence on the delivery of care. Journal of Advanced Nursing, 32(1), 208-214.

Hayes, J. R., \& Flower, L. S. (1980). Identifying the organization of writing processes. In L.W. Gregg $\&{ }^{*}$.R. Steinberg (“ ds.), Cognitive processes in writing (pp. 3-30). Hillsdale (NJ): Lawrence "rlbaum Associates.

Heath, C., \& Luff, P. (1994). Activité distribuée et organisation de l'interaction. Sociologie du travail, 36(4), 523-545.

Hutchins, ". . (1995). Cognition in the wild. Cambridge: The MIT Press.

Kellogg, R.T. (1996). A model of working memory in writing. In C.M. Levy \& S. Ransdell (“ ds.), The science of writing: Theories, methods and applications (pp. 57-72). Mahwah (NJ): Lawrence . rlbaum Associates.

Kerguelen, A. (1991). Pre and post observational categorisation, application to the kronos software aid for activity analyses. In Y. Quéinnec \& F. Daniellou (“ ds.), Designing For Everyone (pp. 504506). London: Taylor \& Francis.

Lardner, R. (1996). " ffective shift handover - A literature review. Offshore technology report - OTO 96003, HSE.

Le Bris, V. (2010). La continuité des activités de production dans des systèmes postés discontinus : 
Approche ergonomique des activités de relève de poste de l'encadrement de proximité. Thèse de doctorat, Université de Toulouse, Toulouse.

Le Bris, V., Barthe, B., Marquié, J.C., Kerguelen, A., Aubert, S., \& Bernadou, B. (2012). Advantages of shift changeovers with meetings: " rgonomics analysis of shift supervisors'activity in aircraft building. Applied Ergonomics, 43(2), 447-454.

Leplat, J. (1993). “ rgonomie et activités collectives. In F. Six \& X. Vaxevanoglou (“' ds.), Les aspects collectifs du travail (pp. 7-28). Toulouse: Octarès.

Leplat, J., \& Savoyant, A. (1983). Ordonnancement et coordination des actions dans les travaux individuels et collectifs. Bulletin de Psychologie, 37(364), 271-278.

Létondal, A.-M. (1997). L'encadrement de proximité : Quels rôles dans les changements d'organisation. Lyon: ANACT.

Maggi, B. (1996). La régulation du processus d'action de travail. In P. Cazamian, F. Hubault \& M. Noulin (“'ds), Traité d'ergonomie (pp. 639-658). Toulouse: Octarès.

Mascia, F. (2001). Gérer dans et avec l'atelier : une approche ergonomique du travail de la maîtrise dans le secteur industriel de production à grande échelle. Thèse de doctorat," PH“ , Paris.

Maury, P., \& Quéinnec, Y. (1993). Qualitative changes in recall memory during day and night shifts. Ergonomics, 36, 289-297.

Moatty, F., Rouard, F., \& Teiger, C. (2004), Les temps de l'écrit dans les nouvelles formes d'organisation du travail. In collectif GRIOT, Figures du temps - Les nouvelles temporalités du travail et de la formation (pp. 149-167). Paris: L'Harmattan.

National Transportation Safety Board. (1992). Aircraft Accident report : In-Flight Structural Breakup, Britt Airways, Inc. dba Continental “ xpress Flight 2574,“" MB-120RT, N33701, “ agle Lake, Texas, September 11, 1991. NTSB/AAR-92/04. Consulté le 12 décembre 2009 de http://www.fss .aero/accident-reports/dvdfiles/US/1991-09-11-US.pdf

Parke, B., \& Kanki, B. (2008). Best Practices in Shift Turnovers: Implications for reducing Aviation maintenance Turnover " rrors as Revealed in ASRS Reports. International Journal of Aviation Psychology, 18(1), 72-85.

Perry, S.J. (2004). Transitions in care: Studying safety in emergency department signovers. Patient Safety, 7(2), 1-3.

Quéinnec, Y., Teiger, V., \& Terssac, G., de. (2008). Repères pour négocier le travail posté (1 $1^{\text {ère }}$ édition : 1992). Toulouse: Octarès.

Rabardel, P. (1995). Les hommes et les technologies : Approche cognitive des instruments contemporains. Paris: Armand Collin.

Rabardel, P., Rogalski, J., \& Béguin, P. (1996). Les processus de coopération à l'articulation entre modalités organisationnelles et activités individuelles. In G. de Terssac, \& “ . Friedberg (" ds.), Coopération et conception (pp. 289-306). Toulouse: Octarès.

Rogalski, J., \& Langa, P. (1997). Activités des cadres et propriétés des situations : Comparaison de deux sites en France et au Zaïre. Le Travail Humain, 60(3), 273-297.

Salembier, P., \& Zouinar, M. (2005). Pas de coopération sans partage ! Le partage d'information comme régulateur de la cognition individuelle et collective. In F. Jeffroy, J. Theureau \& T. Haradji (“ ds.), Relation entre activité individuelle et activité collective : Confrontation de différentes démarches d'études (pp. 55-75). Toulouse: Octarès.

Savoyant, A. (1985). Conditions et moyens de la coordination interindividuelle d'opérations sensorimotrices. Le Travail Humain, 48(1), 59-79.

Sperandio, J.-C. (1975). Compléments à l'étude de la mémoire opérationnelle. Le Travail Humain, $38(1), 41-62$.

Terssac, G. de, \& Chabaud, C. (1990). Référentiel opératif commun. In J. Leplat, \& G. de Terssac (“'ds.), Les facteurs humains de la fiabilité (pp. 111-139). Marseille: Octarès.

Trouvé, P. (1997). Les agents de maîtrise à l'épreuve de la modernisation industrielle. Paris: L'Harmattan.

Tulving, "“ . (1972). " pisodic and semantic memory. In “ . Tulving \& W. Donaldson (“ ds.), Organisation of Memory (pp. 381-402). New York: Academic Press.

Walker, C. R., Guest, R. H., \& Turner, A. N. (1956). The foreman on the assembly line. Cambridge: 
Harvard University Press.

Wilson, S., Galliers, J., \& Fone, J. (2005). Medical handover: a study and implications for information technology. Proceedings of Healthcare systems, Ergonomics and Patient Safety 2005, Florence, Italy, April.

\section{RESUME}

La relève de poste, généralement considérée comme la période de remplacement d'une équipe par une autre, participe à assurer la continuité et la fiabilité du travail posté. Cet article considère la relève de poste de l'encadrement de proximité comme une activité inscrite dans la continuité du travail mise en œuvre au cours des postes successifs. L'étude porte sur le travail de chefs d'équipe dans une situation d'assemblage final en aéronautique travaillant en horaires discontinus $(2 \times 8)$, où des relèves avec rencontre entre deux équipes successives (avec transmissions écrites et orales) alternent avec des relèves sans rencontre (transmissions écrites uniquement). L'analyse centrée sur les activités de rédaction et d'utilisation des écrits de relève de poste dans la continuité du travail posté (avant, pendant et après le changement d'équipes) montre: que la rédaction des écrits s'effectue tout au long des postes quelle que soit la relève à venir, mais qu'elle est systématique à l'approche d'une relève sans rencontre; que les écrits de ce qui est à faire par l'opérateur montant et de ce qui a été fait par le descendant supportent et tracent la coordination de l'activité collective. Ces résultats permettent: de considérer la tâche de relève de poste comme une tâche transversale aux autres tâches de l'encadrement de proximité qui participe à la coordination du travail au cours des postes successifs ; de proposer des principes pour l'aménagement et l'optimisation des relèves de poste.

\section{MOTS CLES}

horaires atypiques, encadrement de proximité, travail posté, communications, travail collectif

\section{RÉFÉRENCEMENT}

Le Bris, V., \& Barthe, B. (2013). Écrits de relève de poste : une activité continue. Activités, 10(1), 31-54, http://www .activites.org/v10n1/v10n1.pdf

Article soumis le 18 mars 2012, accepté pour publication le 26 septembre 2012. 